\title{
Predicting Clinical Variable from MRI Features: Application to MMSE in MCI
}

\author{
S. Duchesne ${ }^{1}$, A. Caroli ${ }^{2}$, C. Geroldi ${ }^{2}$, G.B. Frisoni ${ }^{2}$, and D. Louis Collins ${ }^{1}$ \\ 1 Montréal Neurological Institute (MNI), McGill University, Montréal, Canada \\ ${ }^{2}$ IRCC San Giovanni di Dio, Fatebenefratelli, Brescia, Italy
}

\begin{abstract}
The ability to predict a clinical variable from automated analysis of single, cross-sectional T1-weighted (T1w) MR scans stands to improve the management of patients with neurological diseases. We present a methodology for predicting yearly Mini-Mental Score Examination (MMSE) changes in Mild Cognitive Impairment (MCI) patients. We begin by generating a non-pathological, multidimensional reference space from a group of 152 healthy volunteers by Principal Component Analyses of (i) T1w MR intensity of linearly registered Volumes of Interest (VOI); and (ii) trace of the deformation fields of nonlinearly registered VOIs. We use multiple regression to build linear models from eigenvectors where the projection eigencoordinates of patient data in the reference space are highly correlated with the clinical variable of interest. In our cohort of 47 MCI patients, composed of 16 decliners, 26 stable and 5 improvers (based on MMSE at 1 yr follow-up), there was a significant difference $(P=0.0003)$ for baseline MMSE scores between decliners and improvers, but no other differences based on age or sex. First, we classified our three groups using leave-one-out, forward stepwise linear discriminant analyses of the projection eigencoordinates with $100 \%$ accuracy. Next, we compared various linear models by computing F-statistics on the residuals of predicted vs actual values. The best model was based on 10 eigenvectors + baseline MMSE, with predicted yearly changes highly correlated $(r=0.6955)$ with actual data. Prospective study of an independent cohort of patients is the next logical step towards establishing this promising technique for clinical use.
\end{abstract}

Keywords: MRI, Principal Components Analysis, Intensity, Deformation, Multiple Regression, Mild Cognitive Impairment, Mini-Mental Score Examination.

\section{Introduction}

A number of neurological diseases exhibit pathologically-specific discriminatory information in the form of local intensity variations and shape changes when observed on magnetic resonance images (MRI). The goal of computer-aided diagnosis approaches is to focus and exploit those attributes in order to give physicians a quantitative measurement related to the disease process. Example techniques may perform analysis of the T1-weighted $(\mathrm{T} 1 \mathrm{w})$ signal intensity, serving as an indicator of disease progression, as subtle changes may indicate an underlying 
pathological process before structure integrity is lost. Other approaches will employ co-registration, a process where individual subject images are aligned into a reference space, allowing spatial comparisons to be made between cohorts either at the voxel level, such as in voxel or deformation-based morphometry, or for surfaces. Registration further enables the comparison of individual structures once segmented; manual segmentation and volumetry are considered the gold standard for many research areas. The most promising techniques however move away from single-structure approaches to whole regions of interest, capturing the interrelations between neighboring tissues, and combine individual intensity, texture or registration information, such as in appearance-based approaches [1].

An important and valuable area of research for quantitative MRI analysis resides in the prediction of clinically measured variables. An example of the latter is the Mini-Mental State Examination (MMSE), a cognitive scale to assess normality $(M M S E=30)$, mild impairment $(23 \leq M M S E<30)$ or possible dementia $(M M S E \leq 23) 2$. The assumption is that such neuropsychological or neurological assessments will have a morphological correlate that is detectable via MRI. The ability to categorize cognitive scores from baseline MRI may lead to increased understanding of the disease in question. Further, the ability to predict future scores (i.e., predict change) from baseline MRI is even more important, as it would improve patients management.

\subsection{Mild Cognitive Impairment (MCI)}

MCI is widely viewed as the transition phase between normal aging and Alzheimer's disease (AD) 3], and amnestic MCI individuals are known to be at risk for progression to AD. There is evidence that in those who will progress, measurable hippocampal and entorhinal cortex atrophy, demonstrable on T1w MRI serves as a moderate, though labor-intensive, predictor [4]. Microscopically the strongest predictor of premortem cognitive dysfunction appears to be the relative area of entorhinal cortex occupied by beta-amyloid deposition [5]. Existing MRI measures that have been developped to predict decline are longitudinal, as for example a study by Rusinek et al. [6] showing that an increased rate of atrophy in the MTL predicted future cognitive decline.

In this work we attempt to solve the challenging problem of predicting MMSE changes with a single, cross-sectional MRI measurement. An obvious advantage of having a reliable means of assessing future cognitive decline (within the limits of the MMSE) at baseline, or with one scan, resides in the potentially increased therapeutic effect that comes with earlier detection and treatment. The disadvantage of any cross-sectional approach is that the effect at hand may differ for individuals enrolled in the study or be confounded by another variable, such as aging in the case of MCI. It is important to note that not all MCI patients progress to clinically defined AD, nor show MMSE decline at identical rates 7]. However, careful design and selection of the patient population should serve to improve homogeneity of effect in the test population. Further, we believe that the rate of MMSE decline, along with MTL atrophy, will be linear for MCI patients over the short duration of the study, a reasonable assumption given long-term $\mathrm{AD}$ follow-up data $[8$. 


\subsection{Hypothesis and Goal}

Our general hypothesis is that in the case of neurological pathologies, microscopic changes will be detected via their impact on the T1w MRI signal intensity, while macroscopic alterations in structure shape will be noticed via registration/deformation-based metrics. Consequently, we propose a prediction methodology that (i) uses a large, non-specific Volume of Interest (VOI); (ii) combines intensity and registration-based shape features; and (iii) generates a high dimensional linear model from multiple regression of highly correlated eigenvectors. Our primary goal is to see if we can create such a model, and use MCI patients as a test case of the methodology.

\section{Methods}

Our method can be summarized as follows. First, we generate a non-pathological eigenspace from a large training group of young subjects $(N=152)$. This multidimensional reference eigenspace is created by uniting results from four distinct Principal Component analyses of (i) linearly registered intensity images of the left and right VOIs; and (ii) an approximation of the determinant of the Jacobian matrix of the deformation field within those VOIs. Secondly, patients data are projected in the reference eigenspace and the correlation coefficient between the projection coordinates and the clinical variable is used to identify eigenvectors for the predictive model. The latter is generated via multiple regression against the clinical variable. We compare predictive ability by computing F-statistics based on the residuals of predicted vs actual values for the clinical variable. The methodological details are elaborated in the following sections.

\section{$2.1 \quad$ Subjects}

The Ethics Committee of the Montreal Neurological Institute (Montreal, Canada) and the IRCCS San Giovanni di Dio FBF (Brescia, Italy) approved the study and informed consent was obtained from all participants. A total of 199 subjects were included in this study. The reference group consisted in 152 young, neurologically healthy individuals from the International Consortium for Brain Mapping database (ICBM) 9], whose scans were used to create the non-pathological, reference space. The training population consisted in 47 MCI patients $(23 \leq M M S E<$ 30 ), seen at the IRCCS San Giovanni di Dio FBF Hospital, that have been followed clinically a minimum of 12 months after their initial MR scan.

\subsection{Preprocessing}

MRI data for our 152 ICBM subjects was collected with a T1w MRI protocol on a $1.5 \mathrm{~T}$ scanner (Philips Gyroscan, Best, Netherlands) using a fast gradient echo sequence $\left(T R=18 \mathrm{~ms}, T E=10 \mathrm{~ms}, 1 \mathrm{NEX}\right.$ pulse sequence, flip angle $=30^{\circ}$, matrix size $=256 \times 256, F O V=256 \mathrm{~mm}$, slice thickness $=1 \mathrm{~mm})$. Data for MCI patients were acquired on a $1.0 \mathrm{~T}$ scanner (Philips Gyroscan, Best, Netherlands) using an FFE sequence $(T R=19.7 \mathrm{~ms}, T E=6.9 \mathrm{~ms}$, sagittal acquisition, 
$\left.0.9365 \times 0.9375 \times 1.3 \mathrm{~mm}^{3}\right)$. All global MRI data were processed to correct for intensity non-uniformity due to scanner variations [10. The 152 ICBM subjects were registered in a Talairach-like stereotaxic space in the context of the ICBM project [9]. Most (33/47) of the MCI data were linearly registered (9 DoF) automatically into stereotaxic space [1] while the remaining volumes were manually registered due to high scalp brightness. All reference and training volumes were resampled onto a $1 \mathrm{~mm}$ isotropic grid [11.

Two VOIs were selected for this study, centered on the left and right medial temporal lobe, using Talairach coordinates (start coordinates $x=[-57,+2]$ for the left and right side respectively, $y=-53$ and $z=-52$ ). Each VOI measured $n=55 \times 82 \times 80=360800$ voxels. The VOI was selected so that its extent captured the hippocampus and neighboring MTL structures (e.g. ento and perirhinal cortex, parahippocampal gyrus), irrespective of normal inter- and intra-individual variability. After extraction, each VOI was linearly registered (9 DoF) to the reference volume to further reduce local distortions, and its mean intensity scaled to the mean intensity of the reference VOI, which serves to eliminate the first-order drift in signal measurement between patients.

\subsection{Multi-dimensional Reference Space and Model Creation}

Two image features at each voxel location were retained. The first feature is the grey level intensity consisting in the rasterized data from the intensity-scaled VOIs. The second feature is the trace or the first-order approximation of the determinant of the Jacobian matrix of a non-linear registration-derived deformation field. The latter is calculated to map each subject's VOI to our reference ICBM target. The trace represents an estimate of local volume change. Principal Components Analysis (PCA) is used to reduce the dimensionality of the input training data and generate linear variation models based on the $N=152$ datasets from our ICBM normal subjects. The resulting four PC models were each $p=N-1$ (or 151-dimensional). Most of the variation can usually be explained by a smaller number of modes, $l$, where $l<<n$ and $l<p$. We proceded in selecting 535 eigenvectors in total from our four models (left/right intensity/trace VOIs), that accounted to a per-model variance of $99.7 \%$.

Rasterized vectors of the processed VOI intensity and trace data for each test subject are then projected into the training space, and thus form eigencoordinate vectors. While a number of possible features can be calculated on the distribution of the projected data, our predictor is based on the position along the PC axes. The distribution of eigencoordinates along any principal component for a given population is normally distributed as assessed via Shapiro-Wilke statistics. For each eigenvector the correlation of the eigencoordinate distribution with the clinical variable is then computed. We selected a number $q$ of vectors based on an arbitrarily predefined threshold for the correlation coefficient of $r>|0.30|$. A predictive model is then built from those eigenvectors using multiple regression (JMP IN, SAS Institute, Cary, N. Carolina) and the model is then used to predict the future value of the clinical variable of interest. Residuals and correlation are computed between predicted vs actual value, and F-tests are used 
to determine if the models improve the prediction of our clinical variable from MRI features.

\subsection{Experiments}

Four experiments were completed. Experiment 1 served as a baseline for the classification of our patient population into 3 groups based on their MMSE changes at 1 year follow-up from clinical variables (age, sex, baseline MMSE). Experiment 2 attempted the same 3-group classification but this time based on the projection eigencoordinates in the reference space. Experiment 3 served as a baseline for the prediction of yearly MMSE decline by building a linear model based on clinical variables ("Clinical"). Experiment 4 attempted the same prediction but with a model based on projected eigencoordinates, as per the methodology described above ("MRI"), while in Experiment 5 we added baseline MMSE as an additional variable to the projected eigencoordinates ("MRI+baseline MMSE").

\section{Results}

When comparing MMSE results between baseline and 12 months follow-up, we can separate the 47 patients in the test population into three distinct groups: 16 decliners ( $>-1$ point negative change in MMSE or cognitive decline), 5 improvers ( $>1$ point positive change in MMSE or cognitive improvement), and 26 stable individuals (MMSE change between $[-1,1]$ ). Demographic information about each group can be found in fig. 1. There was no statistically significant age difference between either groups, as assessed from ANOVA and Tukey-Kramer

\begin{tabular}{|c|c|c|c|c|c|}
\hline Groups & \multicolumn{3}{|c|}{ |Decliners Stable Improvers } & & \\
\hline & & & & & \\
\hline Subjects & 16 & 26 & 5 & & \\
\hline & & & & Decliners & \\
\hline $\begin{array}{c}\text { Mean age (yrs) } \\
\text { Std dev }\end{array}$ & $\begin{array}{l}72.4 \\
(4.7)\end{array}$ & $\begin{array}{l}67.6 \\
(8.5)\end{array}$ & $\begin{array}{l}71.8 \\
(5.4)\end{array}$ & & - \\
\hline $\begin{array}{c}\text { Baseline MMSE } \\
\text { Std dev }\end{array}$ & $\begin{array}{l}27.5 \\
(1.3)\end{array}$ & $\begin{array}{l}27.8 \\
(1.4)\end{array}$ & $\begin{array}{l}24.8 \\
(1.8)\end{array}$ & $\operatorname{PC} 40 *$ & Stable \\
\hline $\begin{array}{c}\text { Mean MMSE } \Delta \\
\text { Std dev }\end{array}$ & $\begin{array}{l}-2.9 \\
(1.2)\end{array}$ & $\begin{array}{c}0 \\
(0.8)\end{array}$ & $\begin{array}{c}2.4 \\
(0.6)\end{array}$ & Improvers & \\
\hline
\end{tabular}

Fig. 1. (LEFT) Demographic information. (RIGHT) Leave-one-out, forward stepwise linear discriminant analysis of the patient eigencoordinates in the reference space was $100 \%$ accurate at classifying groups (decliners, stable, improvers). The data is shown here projected on the 3 most discriminating eigenvectors. Our goal was to find an independent basis for the classification and creation of a predictive model, rather than the optimal reference space in which to represent our population. 
HSD $(P>0.05, D F=2)$. There was a statistically significant baseline MMSE difference between the decliners and improvers $(P=0.0003, D F=2)$, but no other significant difference between groups for baseline MMSE. The improvers had the lowest mean baseline MMSE of all three groups.

The classification is based on a leave-one-out, forward stepwise linear discriminant analyses (SYSTAT 10.2, Georgia, PA; P-to-enter $<0.05$ ) of either clinical variables (age, sex and baseline MMSE) or eigencoordinates along the 535 reference space eigenvectors. The clinical classifier of Experiment 1 was $53 \%$ accurate in separating the 47 patients into decliners, improvers and stable subjects $(\mathrm{DF}=2$, Wilk's $\lambda=0.69$ ), while the 3 -way classifier based on projection eigencoordinates of Experiment 2 was $100 \%$ accurate, with 31 significantly discriminant eigenvectors $(P-$ to - enter $<0.05, \mathrm{DF}=31$, Wilk's $\lambda=0)$. Fig. 1 displays the data plotted along the three most discriminating eigenvectors.

While Experiments 1 and 2 classified the data into groups, in the following 3 experiments our goal was to predict the magnitude of the yearly MMSE change.

Table 1. Results from prediction models

\begin{tabular}{c|ccccccccc} 
Model & Features & $\mathrm{r}$ & $r^{2}$ & $\mathrm{SD}$ & $\begin{array}{c}\mathrm{F} \text { stat } \\
\text { to Clin. }\end{array}$ & $\begin{array}{c}\mathrm{P} \\
\text { F stat } \\
\text { to MRI }\end{array}$ & $\mathrm{P}$ \\
\hline Clinical & 3 & 0.429 & 0.176 & 1.86 & - & - & - & - \\
MRI & 10 & 0.668 & 0.446 & 1.53 & 2.499 & 0.003 & - & - \\
MRI+MMSE & 11 & 0.696 & 0.484 & 1.48 & 2.691 & 0.002 & 2.585 & 0.002
\end{tabular}

(A)

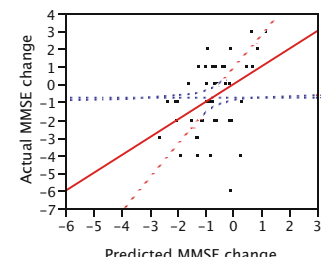

Predicted MMSE change

(B)

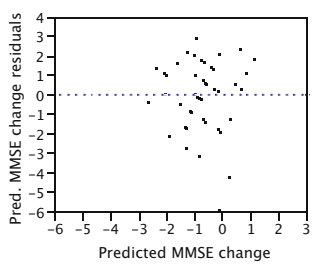

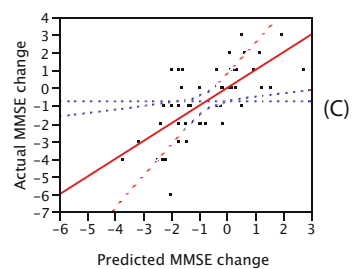

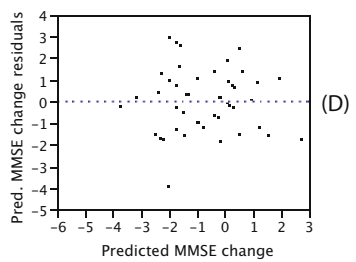

Fig. 2. (A) Clinical model built from multiple regression of age, sex and baseline MMSE against 1 year MMSE changes. (B) Residuals for the "Clinical" model. The correlation of predicted $v s$ actual values was $r=0.429$. (C) MRI+Baseline MMSE model built from multiple regression of the 10 most correlated reference space eigenvectors plus baseline MMSE. (D) Plot of residuals. With a correlation between predicted vs actual yearly MMSE changes of $r=0.6955$, this model was a significant improvement over the "Clinical" one $(F-$ stat $=2.691, P=0.002)$. 
Baseline MMSE, age and sex were all negatively and weakly correlated with 1 year MMSE change $(r=-0.25, r=-0.21$ and $r=0.15$, respectively). In contrast, out of the 535 reference space eigenvectors, 10 had a correlation ratio of $r>|0.30|$. We predicted MMSE change for all patients using each linear model. The number of input features to the model, the resulting correlation $(r)$ and squared correlation $\left(r^{2}\right)$ of predicted vs. actual values, the standard deviation of the predicted score and F-test values (against "Clinical" and "ICBM") are shown in table 1. Recall that the first predictive model (Experiment 3) is based on the 3 clinical variables ("Clinical"), the second (Experiment 4) on the 10 selected eigenvectors ("MRI") and the last (Experiment 5) using the 10 eigenvectors plus the baseline MMSE ("MRI+baseline MMSE"). The linear fit for the "Clinical" and "MRI+baseline MMSE" models are shown in fig. 2, alongside their residual plots. The best model was the "MRI+baselineMMSE" of Experiment 5, with a correlation between predicted and actual value of $r=0.6955$. It was also significantly better than either the "Clinical" model $\left(F\right.$ stat $\left.=3.39, P=0.0001, D F_{1}=43, D F_{2}=35\right)$ or the "MRI" model (Fstat $=$ $\left.2.59, P=0.002, D F_{1}=36, D F_{2}=35\right)$.

\section{Discussion}

Our goal was to demonstrate the application of a generic, multidimensional reference space from MR features for the classification and prediction of a clinical variable. We proceded with analysis of a cohort of 47 Mild Cognitive Impairment patients and achieved 100\% classification accuracy with 31 eigenvectors. We succeeded in creating an 11-variable linear model that predicted yearly MMSE changes, explaining $48.4 \%$ of the variability in the actual data.

Our approach has been to create a model eigenspace based on subjects from the ICBM database, in which we projected our MCI patients. While such a space built from young, neurologically healthy individuals may not be optimal to represent the MCI cohort, it should be noted that our primary goal was to find an independent basis for the creation of a predictive model, and not to find the best representation (mathematical, clinical or otherwise) of the patient population. As the training set is composed of ICBM subjects that are separate from the test set subjects, there is no issue of overdetermination in the creation of the reference space from PCA of the ICBM data. If we are to use this technique for a prospective or retrospective study, we will need either to use a separate test set or to use a leave-one-out technique in order for our regression model to remain independent of any and all training data.

The use of intensity features from MRI raises the question of calibration and normalization. Absolute intensities are rarely used in MRI, since they vary with machine calibration, shimming, and patient-induced variations. We have tried to limit those variations by (1) using the same scanner within groups in the study; (2) ensuring that the same quality assurance procedures were followed for each acquisition at each site; (3) by acquiring subject scans in random group order; and (4) scaling with respect to the reference image. 
Not all macroscopic changes will be capture by the registration process. Point homology in nonlinear registration is of course approximate: in regions where there is complete homology, the displacement field will be nearly exact; and in regions where it is not, the result will be noisy. This uncertainty however is rejected in the PCA model, as it is uncorrelated and non-covarying, and therefore only changes associated with the pathology should remain.

While the classification procedure ought to be tested for generalizability in another set of patients, the leave-one-out classification test (Experiment 2) indicates that it is possible to predict 1-year MMSE changes based on a single MRI scan. This has important ramifications for patient treatment, since success for most therapies is expected to increase with earlier detection of cognitive changes and appropriate treatment. Furthermore, the "MRI + baseline MMSE" linear model (Experiment 5) yields an estimate of the amount of change expected in MMSE over the course of one year. Such data could be used to better taylor therapy for specific patients.

\section{Conclusion}

The ability to perform both classification and prediction of a clinical variable from a single, cross-sectional T1w MRI scan stands to benefit tremendously physicians in the management of patients with MCI. We have explained our methodology for model creation and demonstrated its application to the prediction of yearly MMSE changes in a cohort of MCI subjects. A prospective study on an independent cohort of patients is the next logical step towards further acceptance of this technique in the medical community.

Acknowledgments. Drs. Mazziotta and Evans (ICBM) for permission to use data.

\section{References}

[1] T.F. Cootes et al., IEEE PAMI, 23:6, 2001.

[2] J.R. Petrella et al., Radiology, 226:315-336, 2003.

[3] C. DeCarli et al., Neurology, 63:220-7, 2004.

[4] L.deToledo-Morrell et al., Neurobiol. Aging, 25(9):1197-1203,2004.

[5] B.J. Cummings et al., Neurobiol. Aging, 17:921-33, 1996.

[6] R. Rucinek et al., Radiology, 229:691-96, 2003.

[7] M. Grundman et al., J Mol Neurosci, 19:1-2, 2002.

[8] D. Chan et al., Lancet, 362:1121-2, 2003.

[9] J. Mazziotta et al., NeuroImage, 2:89-101, 1995.

[10] J. Sled et al., IEEE TMI, 17:87-97, 1998.

[11] D. Collins et al., IJPRAI, 11:1271-1294, 1997. 